\title{
Investigation of spontaneous non-linear peptidization dynamics and mechanism with selected $\alpha$-amino acid pairs
}

\author{
Anna Maciejowska ${ }^{1}$ - Agnieszka Godziek ${ }^{1}$. \\ Mieczysław Sajewicz ${ }^{1} \cdot$ Teresa Kowalska $^{1}$
}

Received: 7 November 2015/ Accepted: 15 December 2015/Published online: 22 December 2015

(C) The Author(s) 2015. This article is published with open access at Springerlink.com

\begin{abstract}
The goal of this study was to provide experimental evidence on the dynamics and mechanism of spontaneous oscillatory peptidization in an abiotic system with three $\alpha$-amino acid pairs ( $L$-Met- $L$-Ser, $L$-His- $L$-Thr, and $L$-Cys- $L$-Phg), and to discuss these data in the context of an earlier established theoretical model. For each individual $\alpha$-amino acid in a monocomponent and binary system, the dynamics of peptidization was traced with aid of the high performance liquid chromatograph with the evaporative light scattering detector. As an auxiliary technique, mass spectrometry (MS) was employed to scrutinize structures of the resulting peptides. With $L$-Met- $L$-Ser and $L$-His- $L$-Thr, the dynamics of one amino acid ( $L$-Met and $L$-Thr, respectively) dominated over that of its counterpart. With $L$ Cys- $L$-Phg, no such predominance of the dynamics of one $\alpha$-amino acid over that of its counterpart was observed. Mass spectrometric results confirmed the formation of heteropeptides with each investigated $\alpha$-amino acid pair. With $L$-Met- $L$-Ser, $L$-His$L$-Thr, and $L$-Cys- $L$-Phg, synchronization of the oscillatory behavior in the binary systems was observed, witnessing to mutual cross-catalysis of the two counterparts, assumed by case 4 of the theoretical model.
\end{abstract}

Keywords Binary alpha-amino acid systems - Spontaneous peptidization dynamics · Abiotic amino acid solutions · High performance liquid chromatography · Mass spectrometry

Teresa Kowalska

teresa.kowalska@us.edu.pl

1 Institute of Chemistry, University of Silesia, 9 Szkolna Street, 40-006 Katowice, Poland 


\section{Introduction}

The research on spontaneous oscillatory chiral conversion of the low molecular weight carboxylic acids was initiated over a decade ago with a paper demonstrating this phenomenon upon an example of $S(+)$ ibuprofen [1] and later it was confirmed as a general phenomenon with a selection of other compounds from the groups of profen drugs, hydroxyl acids and amino acids (e.g., [2-4]). Then it was found out that spontaneous oscillatory chiral conversion of the aforementioned compounds is accompanied by spontaneous oscillatory condensation of the same compounds and the two processes are running in the parallel [5-7]. Papers [1-7] furnish general information on the possible molecular mechanisms of these two oscillatory processes, which involve a non-chiral enol/enolate ion intermediary step and can be illustrated by the scheme given in Fig. 1 (for the sake of this study, illustrating the case of amino acids).

Although the experiments presented in papers [1-4] were the first ones to demonstrate spontaneous chiral oscillations, this phenomenon had been assumed earlier by Stich et al. [8] in their theoretical model. Chiral oscillations were directly anticipated in a theoretical activation-polymerization-epimerization-depolymerization (APED) model by Plasson et al. [9], whereas some other models [10-12] had to be modified or reinterpreted in order to account for chiral, rather than just mere chemical oscillations.

In paper [13], the high performance liquid chromatographic results were presented on spontaneous peptidization dynamics for $L$-Pro, $L$-Hyp, and $L$-Pro- $L$ Hyp in $70 \%$ aqueous methanol. It was demonstrated that in the monocomponent amino acid solutions, each compound underwent peptidization with its own dynamics, whereas in a binary solution, both amino acids followed the predominant peptidization dynamics of $L$-Pro. Based on these experimental results, a theoretical model was developed which assumed four different peptidization cases in the binary amino acid mixtures. Case 1 does not assume any cross-catalysis between the two amino acids in a binary solution, so that the peptidization products can only be homopeptides derived from each individual amino acid. Cases 2 and 3 anticipate one or another amino acid apt to cross-catalysis, respectively, and the formation of the homo- and heteropeptides in the system. Case 4 assumes cross-catalysis of both partners and formation of the homo- and heteropeptides also. It can be distinguished from the remaining cases $1-3$ by the unique synchronization of the oscillatory

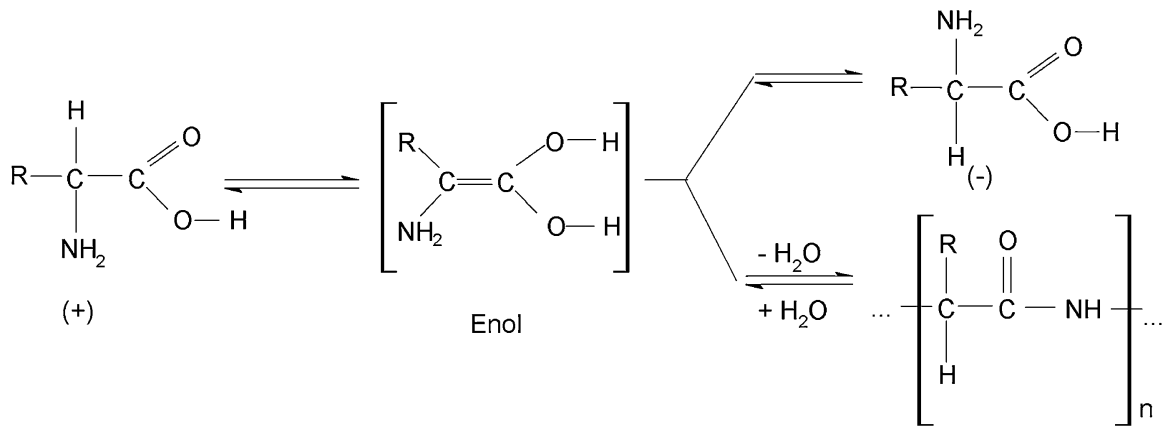

Fig. 1 The parallel processes of chiral conversion and peptidization of an amino acid 
behavior of both partners in a binary system. Recently, a striking finding related to the spontaneous non-linear peptidization of amino acids was described in papers $[14,15]$, namely pulsating changes of the insoluble and hence precipitated higher peptides, which can be perceived even with human eye and instrumentally traced with use of turbidimeter operating in the continuous registration mode.

Continuing our investigations on the peptidization dynamics of $\alpha$-amino acids in the abiotic binary mixtures, in this paper, we present new experimental results obtained for the three randomly selected $\alpha$-amino acid pairs ( $L$-Met- $L$-Ser, $L$-His- $L$ Thr, and $L$-Cys- $L$-Phg). Chemical structures and molecular weights (MW) of the amino acids involved are given in Table 1. The main objective of this study was to compare the peptidization dynamics in the monocomponent amino acid solutions against those in the binary mixtures, in the context of their respective chemical structures and the earlier developed theoretical model. The main analytical technique to trace peptidization dynamics was high performance liquid chromatography with the evaporative light scattering detection (HPLC-ELSD), and as an auxiliary instrumental technique, mass spectrometry (MS) was used to scrutinize chemical structures of the obtained peptides.

\section{Experimental}

\section{Reagents}

In our experiment, we used $L$-Ser, $L$-Met, $L$-Cys, $L$-Thr and $L$-His (Reanal, Budapest, Hungary), and L-Phg (Sigma-Aldrich, St Louis, MO, USA). All amino

Table 1 Chemical structures and molecular weights (MW) of $\alpha$-amino acids investigated in pairs $L$-Met$L$-Ser, $L$-His- $L$-Thr, and $L$-Cys- $L$-Phg

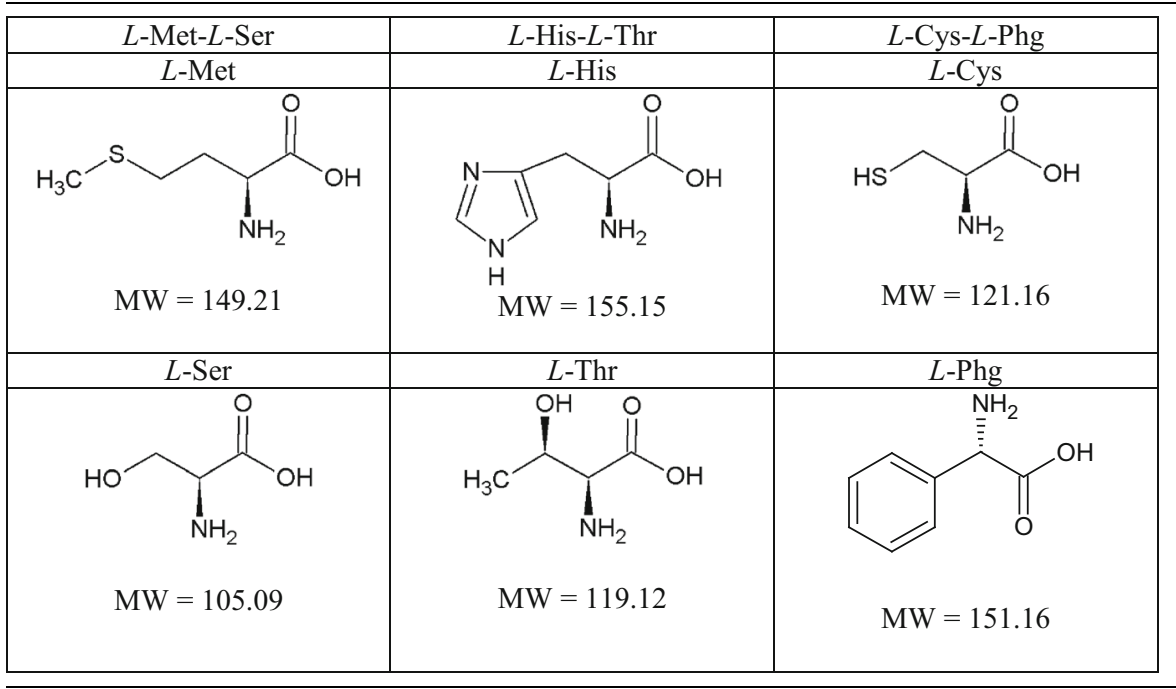


acids were of analytical purity. Methanol and acetonitrile (Sigma-Aldrich) were of HPLC purity. Water was de-ionized and double distilled in our laboratory by means of the Elix Advantage model Millipore System (Molsheim, France).

For the HPLC/ELSD, HPLC/MS, and MS experiments, we prepared the $1.0 \mathrm{mg} \mathrm{mL}{ }^{-1}$ solutions of $L$-Phg, $L$-Thr, $L$-Ser, and $L$-Cys (i.e., $6.61 \times 10^{-3}$ mol L ${ }^{-1}$ for $L$-Phg, $8.39 \times 10^{-3} \mathrm{~mol} \mathrm{~L}^{-1}$ for $L$-Thr, $9.52 \times 10^{-3} \mathrm{~mol} \mathrm{~L}^{-1}$ for $L$ Ser, and $8.25 \times 10^{-3} \mathrm{~mol} \mathrm{~L}^{-1}$ for L-Cys) in $70 \%$ aqueous $\mathrm{MeOH}$, and the $2.0 \mathrm{mg} \mathrm{mL}^{-1}$ solutions of $L$-His and $L$-Met (i.e., $1.29 \times 10^{-2} \mathrm{~mol} \mathrm{~L}^{-1}$ for $L$-His and $1.34 \times 10^{-2} \mathrm{~mol} \mathrm{~L}^{-1}$ for $L$-Met) in $70 \%$ aqueous $\mathrm{MeOH}$. The $70 \%$ aqueous methanol, known for its strong antiseptic properties, was selected in order to protect the amino acid solutions purposely stored for longer periods of time from the microbial action.

\section{HPLC with evaporative light scattering detection}

The condensation process instantaneously commencing in each freshly prepared monocomponent and binary amino acid solution was monitored by means of the achiral HPLC with the ELSD detector. This HPLC mode was employed to separate the oligopeptides from the non-peptidized amino acids and also to fractionate the oligopeptides. The analyses were carried out using the Varian model 920 liquid chromatograph (Varian, Harbor City, CA, USA) equipped with the Varian 900-LC model autosampler, the gradient pump, the Varian 380-LC model ELSD detector, and the Galaxie software for data acquisition and processing. For the analysis of $L$ Met- $L$-Ser and $L$-His- $L$-Thr, the Hypersil C18 (5 $\mu \mathrm{m}$ particle diameter) column $(150 \mathrm{~mm} \times 4.6 \mathrm{~mm}$ i.d.; ThermoQuest; cat. no. 3718-062) was used, and for the analysis of $L$-Cys- $L$-Phg, the Pursuit $5 \mathrm{C} 18$ (5 $\mu \mathrm{m}$ particle diameter) column (250 $\mathrm{mm} \times 4.6 \mathrm{~mm}$ i.d.; Varian; cat. no. A3000250C046) was employed.

All analyses were carried out in the isocratic mode. With $L$-Met- $L$-Ser and $L$-His$L$-Thr, the injected sample aliquots were $3 \mu \mathrm{L}$, and with $L$-Cys- $L$-Phg, the injected sample aliquots were $5 \mu \mathrm{L}$. With the monocomponent $L$-Met and $L$-Ser solutions, and the binary $L$-Met- $L$-Ser mixture, acetonitrile: $1 \%$ aqueous $\mathrm{CH}_{3} \mathrm{COOH}$ (10:90, $v / v$ ) was employed as a mobile phase, at a flow rate of $0.8 \mathrm{~mL} \mathrm{~min}^{-1}$. With the monocomponent $L$-His and $L$-Thr solutions, and the binary $L$-His- $L$-Thr mixture, methanol: $1 \%$ aqueous $\mathrm{CH}_{3} \mathrm{COOH}(50: 50, v / v)$ was employed as a mobile phase, at

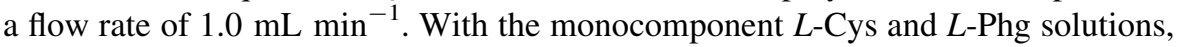
and the binary $L$-Cys- $L$-Phg mixture, methanol:water $(20: 80, v / v)$ was employed as a mobile phase, at a flow rate of $0.8 \mathrm{~mL} \mathrm{~min}^{-1}$. The chromatographic columns were thermostatted at $35{ }^{\circ} \mathrm{C}$ in the Varian ProStar 510 model column oven. The analyses of the $L$-Met- $L$-Ser, $L$-His- $L$-Thr and $L$-Cys- $L$-Phg systems were carried out for $30 \mathrm{~h}$ in the 8-min intervals.

\section{HPLC with mass spectrometric detection}

The high performance liquid chromatographic system described in the preceding section was additionally equipped with the Varian 100-MS mass spectrometric detector and the Varian MS Workstation v. 6.9.1 software for data acquisition and 
processing. The mass spectrometric detection was carried out for the $L$-Met- $L$-Ser solution in $70 \%$ aqueous methanol aged for 9 days. The working MS conditions were the following ones: the ESI mode (ESI-MS scan, negative ionization, spray chamber temperature $50{ }^{\circ} \mathrm{C}$, drying gas temperature $350{ }^{\circ} \mathrm{C}$, drying gas pressure 25 psi, capillary voltage $50 \mathrm{~V}$, needle voltage $5 \mathrm{kV}$ ).

\section{MS}

For the mass spectrometric analyses, we used the Thermo LCQ Deca XP Plus MS system. The analyses were carried out for the binary $L$-His- $L$-Thr and $L$-Cys- $L$-Phg solutions in $70 \%$ aqueous methanol aged for 8 and 1 month, respectively. The working MS conditions were the following ones: the ESI mode (ESI-MS scan, positive and negative ionization, capillary voltage $50 \mathrm{~V}$, needle voltage $5 \mathrm{kV}$, and needle temperature $250{ }^{\circ} \mathrm{C}$ ).

\section{Results and discussion}

A basic requirement to reliably trace concentration changes of individual amino acids in a binary solution as a function of time is to employ a chromatographic system that allows the baseline separation of the amino acid pairs. Moreover, the
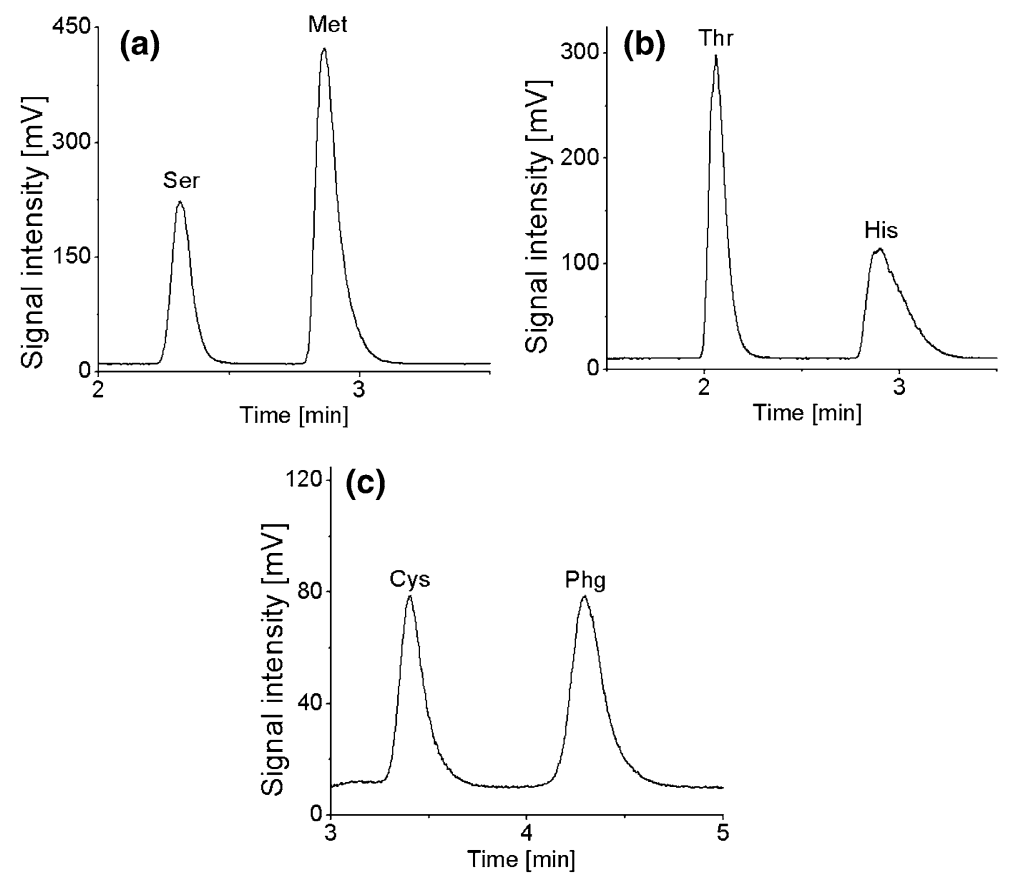

Fig. 2 The base-line separations of the investigated amino acids in the binary mixtures with use of the employed HPLC-ELSD system: a Met-Ser; b His-Thr; c Cys-Phg 
shorter is an individual analytical run, the more detailed kinetic information can be obtained on the dynamics of peptidization. The HPLC-ELSD working conditions assumed in this study fully satisfy the aforementioned requirements. Each chromatographic run was carried out for 8 min only (i.e., sampling was automatically done in the 8-min intervals). The baseline separation of each investigated amino acid pair was obtained, as shown in Fig. 2. The retention times of $L$-Met and $L$-Ser were $3.1 \pm 0.2$ and $2.5 \pm 0.2 \mathrm{~min}$, those of $L$-His and $L$-Thr were $3.1 \pm 0.2$ and $2.1 \pm 0.1 \mathrm{~min}$, and those of $L$-Cys and $L$-Phg were $3.5 \pm 0.1$ and $4.4 \pm 0.1 \mathrm{~min}$.

\section{HPLC-ELSD of $L$-Met, $L$-Ser, and $L$-Met- $L$-Ser}

In the course of the 30-h lasting ageing of the monocomponent $L$-Met and $L$-Ser solutions and the binary $L$-Met- $L$-Ser mixture, the chromatographic peak heights of individual amino acids were changing in a non-linear fashion, unequivocal with the respective non-linear concentration changes (Figs. 3a-3c). Based on the plots given in Figs. 3a-3c, the following observations were made. First, the amplitudes of the oscillatory concentration changes with $L$-Met in the monocomponent solution were
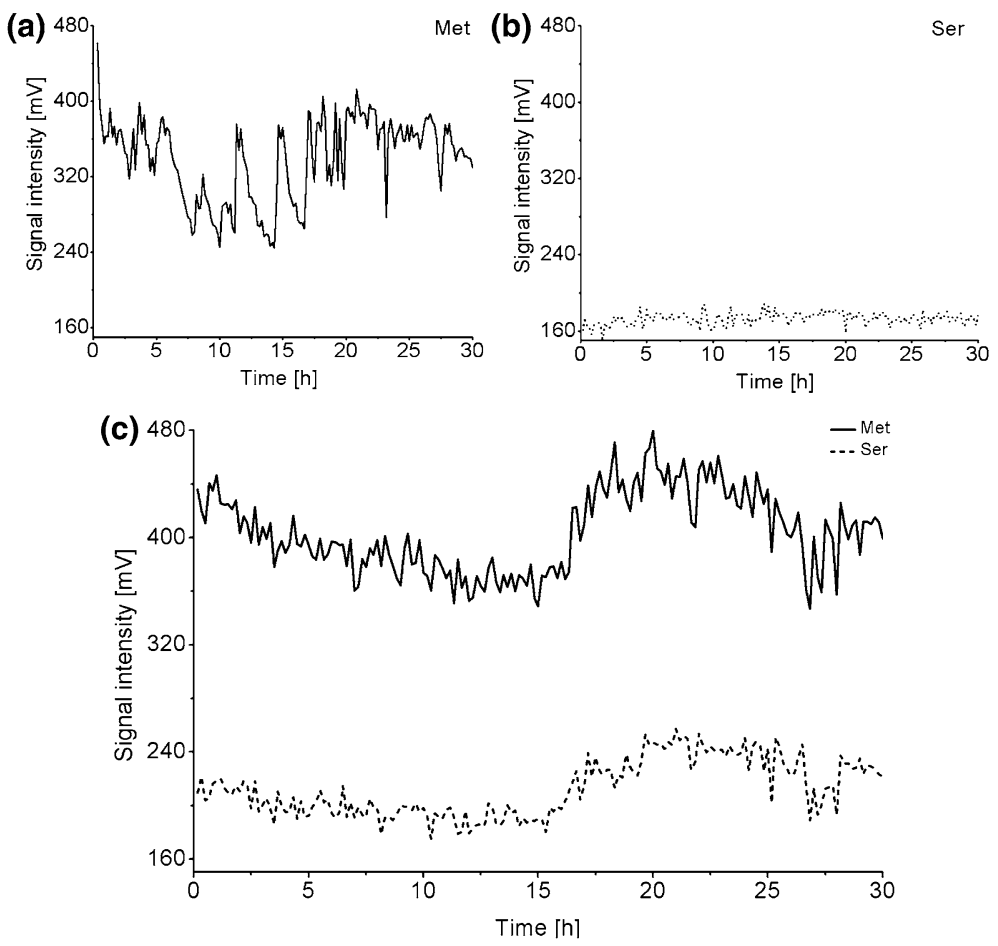

Fig. 3 Time series of the chromatographic peak heights for Met-Ser in $70 \%$ aqueous methanol (registered with ELSD detector): a single Met; b single Ser; c Met and Ser in a binary mixture. The 0-30 h storage period 
in the range of $100 \mathrm{mV}$ signal intensity units (Fig. 3a), whereas with the monomeric $L$-Ser and the binary $L$-Met- $L$-Ser solution, they were by one magnitude order lower (Fig. $3 b$ and c). These results indicate that the peptidization process of $L$-Met in the monocomponent solution characterizes with different dynamics from that in the binary solution. Second, the oscillatory patterns of the time series of the chromatographic peak heights valid for $L$-Met and $L$-Ser in the binary $L$-Met- $L$ Ser system are perfectly synchronized (Fig. 3c), witnessing to mutual crosscatalysis of the two counterparts, assumed by case 4 of the theoretical model [13]. Third, the oscillatory patterns of $L$-Ser and $L$-Met in the monocomponent solutions look different from one another (Figs. 3a and 3b), yet in the binary mixture both of them roughly resemble that of pure $L$-Met (even, if the amplitudes of the concentration changes in the former case are lower than in the latter one). This observation suggests that the dynamics of peptidization in the binary $L$-Met- $L$-Ser solution is controlled by that of $L$-Met (which contains 4 carbon atoms in its aliphatic chain against three carbon atoms of $L$-Ser). Thus, it can be assumed that the higher aliphatic chain length of $L$-Met more effectively hinders intermolecular interactions of the $-\mathrm{NH}_{2}$ and $-\mathrm{COOH}$ functionalities and hence, $L$-Met controls an
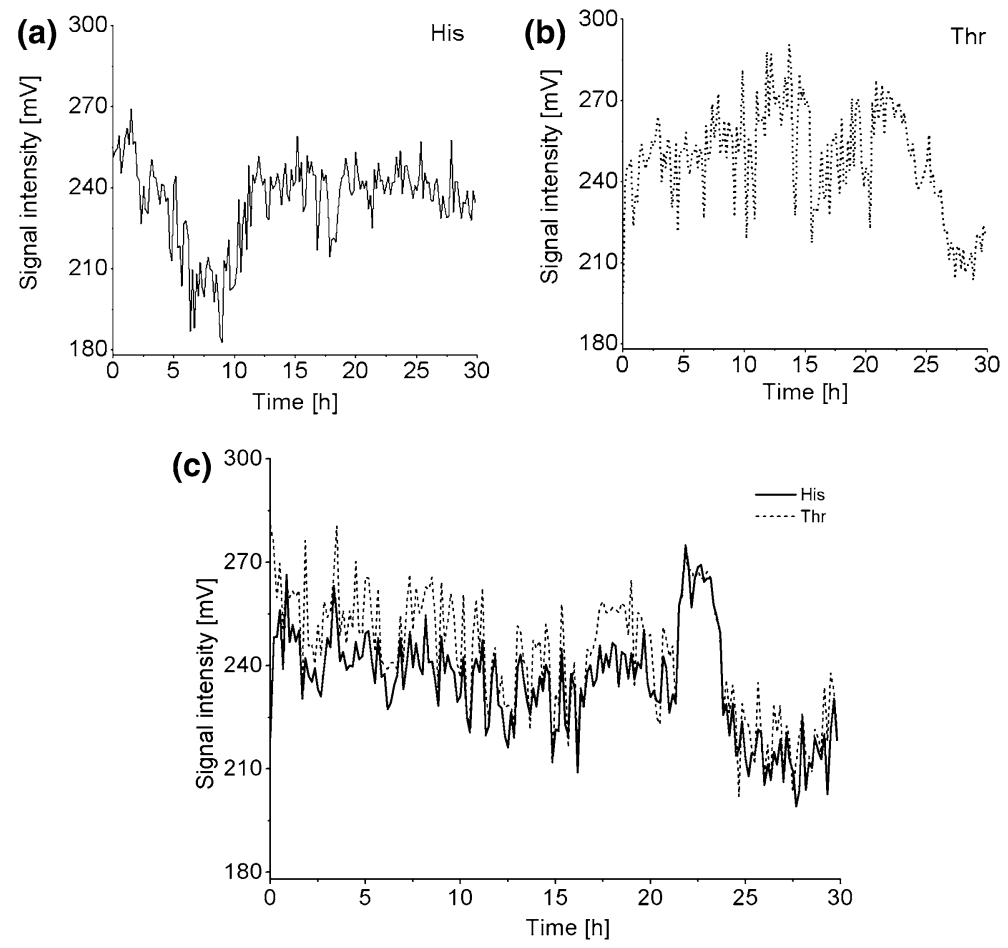

Fig. 4 Time series of the chromatographic peak heights for His-Thr in $70 \%$ aqueous methanol (registered with ELSD detector): a single His; b single Thr; c His and Thr in a binary mixture. The 0-30 h storage period 
overall peptidization dynamics (and more specifically, the rate of the heteropeptides formation).

\section{HPLC-ELSD of $L$-His, $L$-Thr, and $L$-His- $L$-Thr}

The second investigated amino acid pair was $L$-His- $L$-Thr. The time series of the peak heights for $L$-His and $L$-Thr in the monocomponent amino acid solutions and in the $L$-His- $L$-Thr binary system are presented in Figs. $4 \mathrm{a}-4 \mathrm{c}$. Based on these plots, the following observations were made. First, the amplitudes of the oscillatory peak height changes are similar for the amino acids in the monocomponent and the binary systems, and remain in the range of several dozen $\mathrm{mV}$ signal intensity units. Second, the oscillatory patterns of the time series of the chromatographic peak heights valid for $L$-His and $L$-Thr in the binary system are well enough synchronized (Fig. 4c), again witnessing to mutual cross-catalysis of the two counterparts, assumed by case 4 of the theoretical model [13]. Third, the time series patterns valid for $L$-His and $L$ Thr in the monocomponent solutions are not similar (Figs. 4a and 4b), yet in the binary mixture it looks somewhat different (Fig. 4c). Although for the initial 15-h ageing, the oscillatory patterns valid for $L$-His and $L$-Thr do not resemble those for the monocomponent systems, later (i.e., starting from the $15 \mathrm{~h}$ ageing) they start
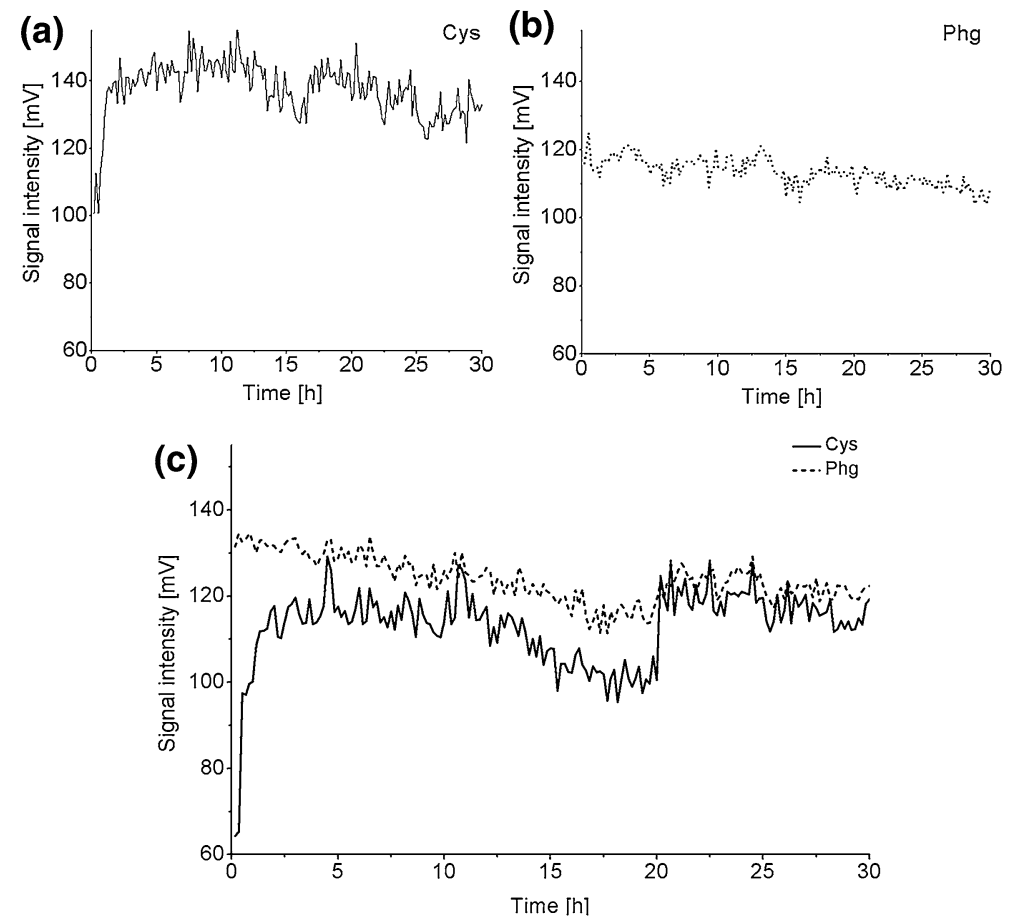

Fig. 5 Time series of the chromatographic peak heights for Cys-Phg in $70 \%$ aqueous methanol (registered with ELSD detector): a single Cys; b single Phg; c Cys and Phg in a binary mixture. The $0-30 \mathrm{~h}$ storage period 
resembling pattern valid for the monomeric $L$-Thr. This observation suggests that starting from that moment, the dynamics of peptidization in the binary $L$-His- $L$-Thr solution becomes controlled by that of $L$-Thr. This predominance of $L$-Thr can be due to four carbon atoms in its aliphatic chain against three carbon atoms of $L$-His. However, $L$-His contains a bulk imidazole group in its structure, also able to obstruct intermolecular interactions of the $-\mathrm{NH}_{2}$ and $-\mathrm{COOH}$ functionalities, and $L$ Thr contains the $-\mathrm{OH}$ group, which allows a competitive condensation pattern through esterification. Probably these are the main reasons why the predominance of the $L$-Thr pattern is not instantaneous.

\section{HPLC-ELSD of $L$-Cys, $L$-Phg, and $L$-Cys- $L$-Phg}

In the course of the 30 -h ageing of the monocomponent $L$-Cys and $L$-Phg solutions and the binary $L$-Cys- $L$-Phg system, the non-linear changes of the chromatographic peak heights with both amino acids were observed, unequivocal with the respective concentration changes (Figs. 5a-5c). Based on these plots, the following observations were made. First, the amplitudes of the $L$-Cys peak height oscillations in the monocomponent system are higher than those of $L-P h g$, and the same observation is valid for the initial ca. $20 \mathrm{~h}$ of the binary system also. In the final $10 \mathrm{~h}$ of the binary sample storage, the oscillatory amplitudes for both amino acids become comparable with each other. Second, starting from the third hour of the binary sample storage, concentration changes of $L$-Cys and $L$-Phg are well enough synchronized (Fig. 5c), witnessing to mutual cross-catalysis of the two counterparts, assumed by case 4 of the theoretical model [13]. Third, the oscillatory patterns of $L$-Cys and $L$-Phg in the monocomponent systems are not similar (Figs. 5a and 5b) and in the binary mixture, none of these two oscillatory patterns predominates. Although the aliphatic chains of $L$-Cys and $L$-Phg contain 3 and 2 carbon atoms, respectively, their condensation abilities are substantially different. $L$-Cys contains not only the $-\mathrm{NH}_{2}$ and $-\mathrm{COOH}$ functionalities in its molecule, but also a reactive thiol $(-\mathrm{SH})$ group, responsible for spontaneous formation of disulfide bridges and hence, for the ternary structure and the spherical shape of the $L$-Cys-derived proteins and peptides $[16,17]$. On the other hand, an aromatic ring in molecular structure of $L$-Phg can partially shield its $-\mathrm{NH}_{2}$ and $-\mathrm{COOH}$ functionalities, thus hampering the peptidization dynamics of $L$-Phg.

\section{HPLC-MS of $L$-Met- $L$-Ser}

The HPLC-MS analysis was performed for the aged $L$-Met- $L$-Ser sample (after 9 days ageing) and its goal was to check, if peptidization spontaneously carried out in this binary system results in both, homo- and heteropeptides (or in homopeptides only). In Fig. 6, we give an HPLC-MS chromatogram and the mass spectra recorded for chromatographic peak 1 (Ser), peak 2 (Met), and peak 3 (the main peptidization product). With peak 1 (Ser), we observe the predominant signal at $\mathrm{m} / z$ 233 , which can be attributed to the homopeptide structure $\left[\mathrm{Ser}_{3}-\mathrm{COOH}-\mathrm{H}\right]^{-}$, yet signal at $m / z 104$ originating from the Ser molecular ion ([Ser-H $\left.]^{-}\right)$is also present. The remaining signals are of low intensity only and they can be attributed to peptides. With peak 2 (Met), signal at $\mathrm{m} / z 148$ originating from the Met molecular 
ion $\left([\mathrm{Met}-\mathrm{H}]^{-}\right)$is present, but signals originating from peptides are predominant. With peak 3 (the main peptidization product), the molecular ions derived from Ser and Met are absent, but a good number of signals originating from heteropeptides can be observed (e.g., signals at $\mathrm{m} / \mathrm{z} 1717$ and 1895, which can be attributed to $\left[\mathrm{Ser}_{12}+\mathrm{Met}_{5}\right]^{-}$and $\left[\mathrm{Ser}_{2}+\mathrm{Met}_{13}\right]^{-}$, respectively). To sum up, peptidization spontaneously running in the $L$-Met- $L$-Ser sample results both, in homo- and heteropeptides.

\section{MS of $L$-His- $L$-Thr and $L$-Cys- $L$-Phg}

Investigations by means of MS were carried out for the aged binary solutions of $L$ His- $L$-Thr and $L$-Cys- $L$-Phg. In Fig. 7 , we show mass spectra recorded for the binary $L$-His- $L$-Thr system in the positive (Fig. 7a) and negative ionization mode (Fig. 7b), respectively. In Figs. 8a and 8b, the analogous mass spectra are presented, valid for the aged $L$-Cys- $L$-Phg system. In all these spectra, signals originating from molecular ions of the investigated $\alpha$-amino acids can be seen (i.e., originating from the $[\mathrm{His}+\mathrm{H}]^{+},[\mathrm{His}-\mathrm{H}]^{-},[\mathrm{Thr}+\mathrm{H}]^{+},[\mathrm{Thr}-\mathrm{H}]^{-},[\mathrm{Cys}-\mathrm{H}]^{-}$, and $[\mathrm{Phg}+\mathrm{H}]^{+}$ion). The most interesting signals are, however, those originating from the spontaneously formed peptides. These signals are abundant and the predominant number thereof originates from heteropeptides, although signals originating from homopeptides are encountered as well. For the sake of example, let us point out to such homopeptides, as $\left[\mathrm{Thr}_{3}+2 \mathrm{H}\right]^{2+}\left(\mathrm{m} / \mathrm{z}\right.$ 160.5; Fig. 7a), and $\left[\mathrm{Cys}_{2}+2 \mathrm{Na}\right]^{2+},\left[\mathrm{Phg}_{8}+2 \mathrm{H}\right]^{2+}$, and $\left[\mathrm{Cys}_{5}+\mathrm{Na}\right]^{+}(\mathrm{m} / \mathrm{z}$ 135.0, 540.2, and 556.0; Fig. 8a). Generally, the observed heteropeptides can be divided into two groups. In one group, an evident quantitative predominance of one amino acid over its counterpart is observed and the following

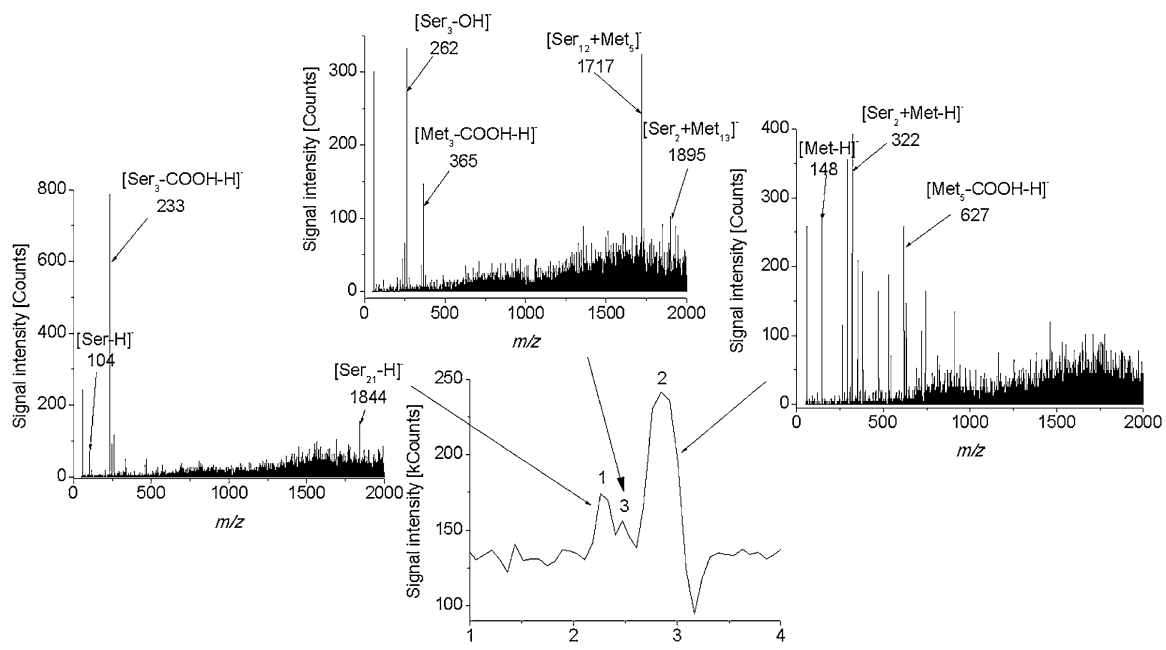

Fig. 6 The HPLC-MS chromatogram recorded for the aged Met-Ser solution in $70 \%$ aqueous methanol and the respective mass spectra recorded for Ser (peak 1), Met (peak 2), and main peptidization product (peak 3) 
(a)

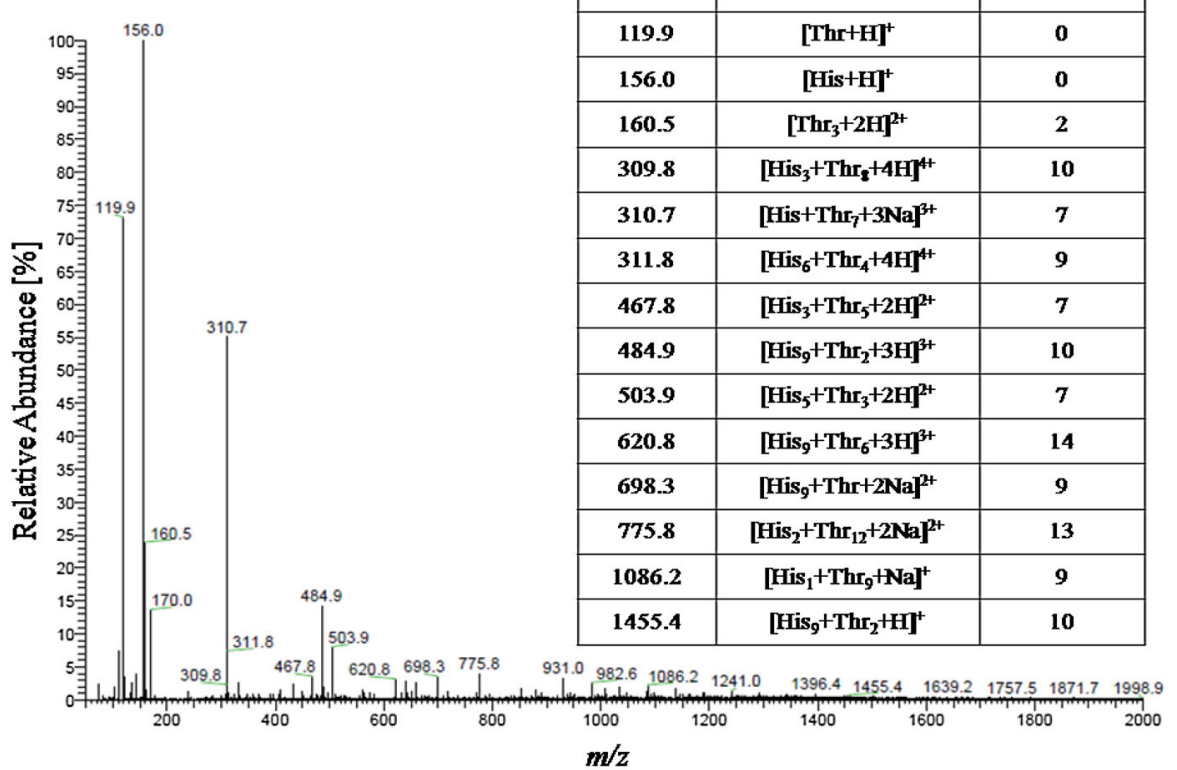

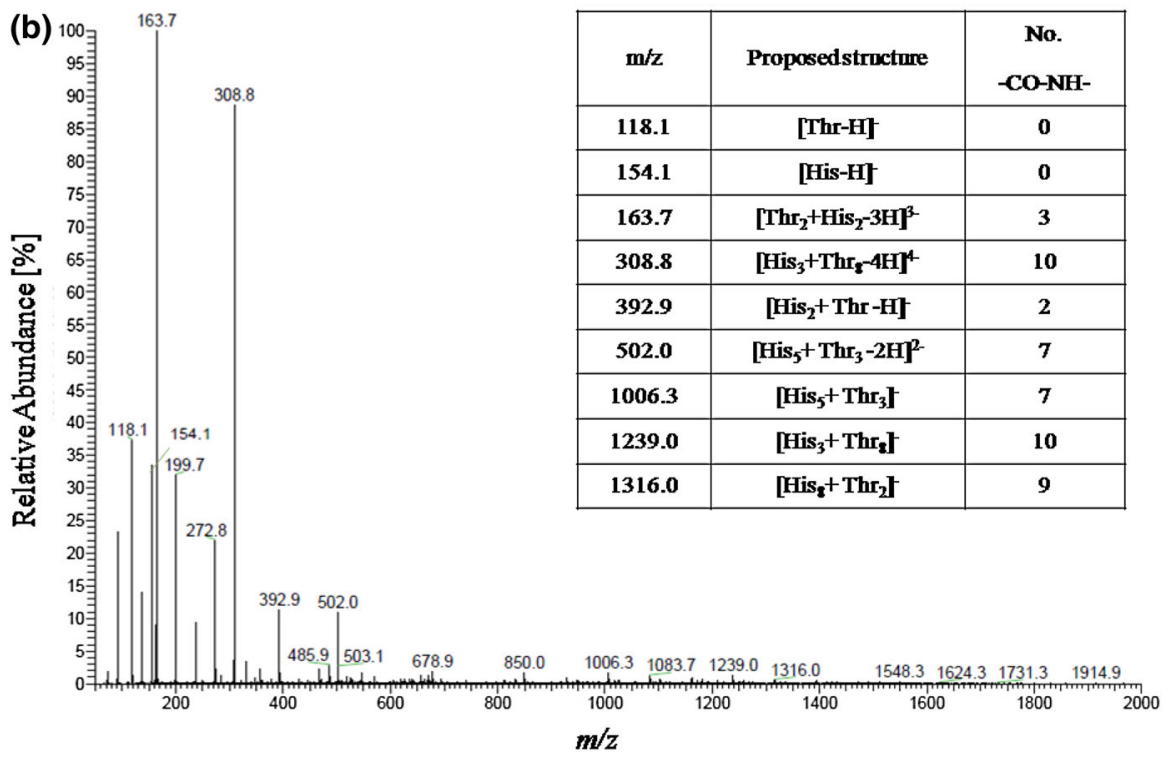

Fig. 7 Mass spectra recorded in the a positive and $\mathbf{b}$ negative ionization mode for the His-Thr solution in $70 \%$ aqueous methanol aged for 8 months, suggested chemical structures of certain cations and anions, and the respective numbers of peptide bonds (-CO-NH-) 
(a)

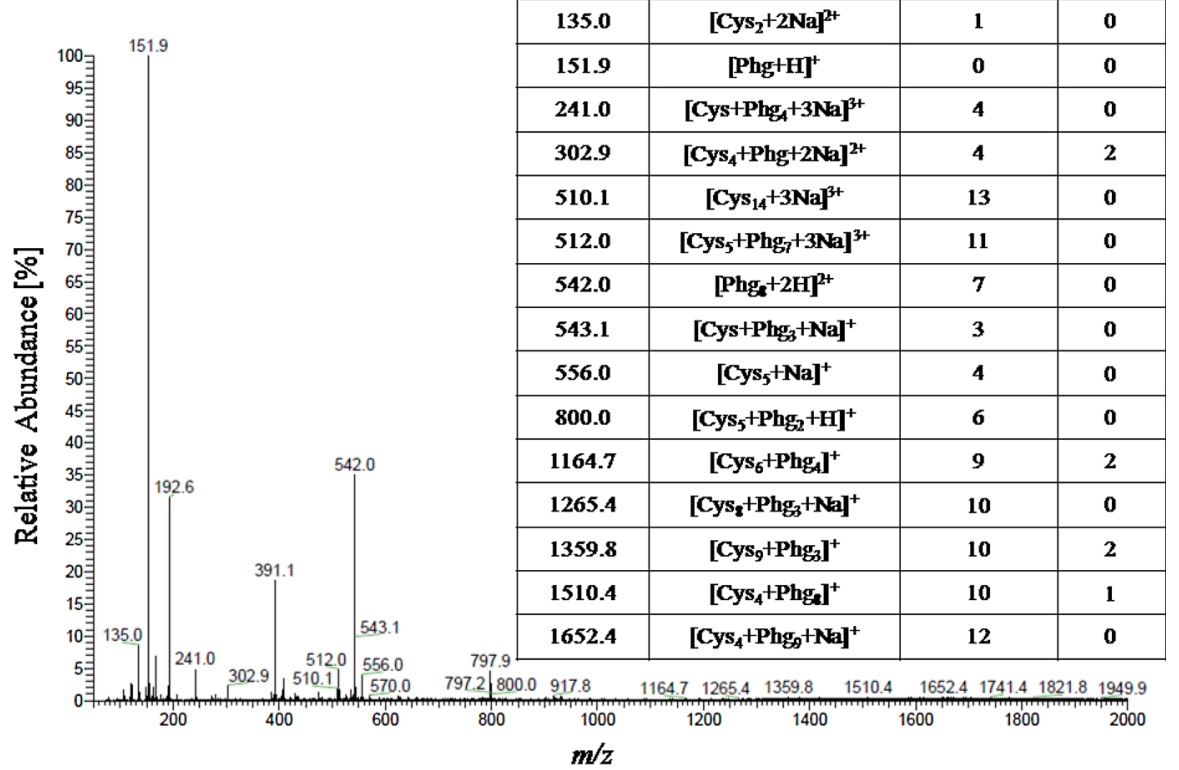

(b)

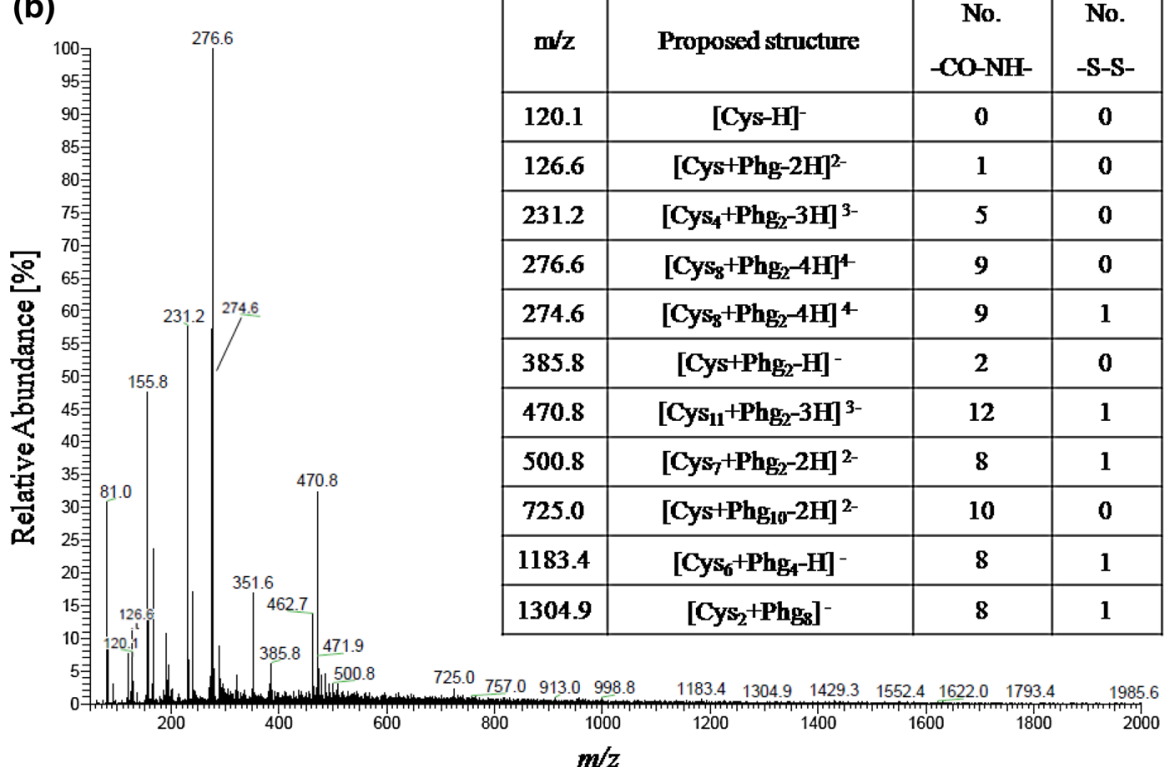

Fig. 8 Mass spectra recorded in the a positive and $\mathbf{b}$ negative ionization mode for the aged Cys-Phg solution in $70 \%$ aqueous methanol aged for 1 month, suggested chemical structures of certain cations and anions, and the respective numbers of peptide bonds $(-\mathrm{CO}-\mathrm{NH}-)$ and disulfide bridges $(-\mathrm{S}-\mathrm{S}-)$ 
signals can be given as an example: $m / z$ 484.9, $\left[\mathrm{His}_{9}+\mathrm{Thr}_{2}+3 \mathrm{H}\right]^{3+} ; m / z$ 775.8, $\left[\mathrm{His}_{2}+\mathrm{Thr}_{12}+2 \mathrm{Na}\right]^{2+} ; m / z$ 308.8, $\left[\mathrm{His}_{3}+\mathrm{Thr}_{8}-4 \mathrm{H}\right]^{4-} ; \mathrm{m} / z$ 1316.0, $\left[\mathrm{His}_{8}+\mathrm{Thr}_{2}\right]^{-}$; $m / z \quad 543.1, \quad\left[\mathrm{Cys}+\mathrm{Phg}_{3}+\mathrm{Na}\right]^{+} ; \quad m / z \quad 1265.4, \quad\left[\mathrm{Cys}_{8}+\mathrm{Phg}_{3}+\mathrm{Na}\right]^{+}, \quad m / z \quad 470.8$, $\left[\mathrm{Cys}_{11}+\mathrm{Phg}_{2}-3 \mathrm{H}\right]^{3-}$; and $\mathrm{m} / z$ 725.0, $\left[\mathrm{Cys}+\mathrm{Phg}_{10}-2 \mathrm{H}\right]^{2-}$. The other group contains heteropeptides in the molecular ratio of $1: 1$ (e.g., $\left[\mathrm{Thr}_{2}+\mathrm{His}_{2}-3 \mathrm{H}\right]^{3-}$ and $[\mathrm{Cys}+\mathrm{Phg}-2 \mathrm{H}]^{2-}$ at $m / z 163.7$ and 126.6, respectively.

\section{Conclusions}

The experimental results presented in this study show that with each investigated $\alpha$ amino acid pair ( $L$-Met- $L$-Ser, $L$-His- $L$-Thr, and $L$-Cys- $L$-Phg), synchronization of the oscillatory concentration changes of the monomeric amino acids is observed, witnessing to mutual cross-catalysis of the two counterparts. These results serve as an experimental confirmation of case 4 of the theoretical model presented elsewhere. Case 4 seems to be the most physical one from the point of view of the law of mass action, because it anticipates intermolecular interactions among all molecules present in a given system. Additional experimental support is provided by the mass spectrometric evidence on the heteropeptide formation with each investigated binary system. An effect of molecular structure of the amino acids involved (and more specifically, the shielding effect of the $-\mathrm{NH}_{2}$ and $-\mathrm{COOH}$ functionalities with aliphatic chains) on an overall dynamics of peptidization is perceptible as well as an influence of the other functionalities present in certain molecules (-OH with $L$-Thr and $-\mathrm{SH}$ with $L$-Cys).

Acknowledgments We thank Prof. Irving R. Epstein for enlightening discussions of the dynamics of amino acid condensation in binary solutions. One author (A.G.) acknowledges the financial support of the DoktoRIS project, co-financed by the European Union within the European Social Found.

Open Access This article is distributed under the terms of the Creative Commons Attribution 4.0 International License (http://creativecommons.org/licenses/by/4.0/), which permits unrestricted use, distribution, and reproduction in any medium, provided you give appropriate credit to the original author(s) and the source, provide a link to the Creative Commons license, and indicate if changes were made.

\section{References}

1. Sajewicz M, Piętka R, Pieniak A, Kowalska T (2005) Acta Chromatogr 15:131-149

2. Sajewicz M, Grygierczyk G, Gontarska M, Kowalska T (2007) J Liq Chromatogr Relat Technol 30:2185-2192

3. Sajewicz M, Kronenbach D, Gontarska M, Wróbel M, Pietka R, Kowalska T (2009) J Planar Chromatogr-Mod TLC 22:241-248

4. Sajewicz M, Gontarska M, Wojtal Ł, Kronenbach D, Leda M, Epstein IR, Kowalska T (2008) J Liq Chromatogr Relat Technol 31:1986-2005

5. Sajewicz M, Gontarska M, Kronenbach D, Berry E, Kowalska T (2012) J Chromatogr Sci 50:237-244

6. Sajewicz M, Matlengiewicz M, Leda M, Gontarska M, Kronenbach D, Kowalska T, Epstein IR (2010) J Phys Org Chem 23:1066-1073 
7. Sajewicz M, Dolnik M, Kronenbach D, Gontarska M, Kowalska T, Epstein IR (2011) J Phys Chem A 115:14331-14339

8. Stich M, Blanco C, Hochberg D (2013) Phys Chem Chem Phys 15:255-261

9. Plasson R, Bersini H, Commeyras A (2004) Proc Natl Acad Sci USA 101:16733-16738

10. Hyver C (1985) J Chem Phys 83:850-851

11. Bykov VI, Gorban AN (1987) Chem Eng Sci 42:1249-1251

12. Peacock-Lopez E, Radov DB, Flesner CS (1997) Biophys Chem 65:171-178

13. Dolnik M, Sajewicz M, Kowalska T, Epstein IR (2014) RSC Adv 4:7330-7339

14. Godziek A, Maciejowska A, Talik E, Wrzalik R, Sajewicz M, Kowalska T (2016) Curr Protein Pept Sci (in press)

15. Maciejowska A, Godziek A, Sajewicz M, Kowalska T (2017) Acta Chromatogr. doi:10.1556/1326. 2017.29.1.00

16. Godziek A, Maciejowska A, Talik E, Sajewicz M, Kowalska T (2015) J Planar Chromatogr-Mod TLC 28:144-151

17. Carny O, Gazit E (2005) FASEB J 19:1051-1055 